cision of the sector scanner developed by the Admiralty Research Laboratory at Teddington, Middlesex. Dr F. R. Harden Jones has liberated tagged plaice at sea and tracked them with the sector scanner as they were trawled. $\mathrm{He}$, and his colleagues from the Lowestoft Laboratory, have tracked and trawled seventy-five fish so far; their results, dramatically recorded on film, are revealing a great deal that was unknown, or speculative, about the behaviour of fish in the vicinity of otter boards, tickler chains, bridles, foot ropes and all the paraphernalia of commercial trawling. Their preliminary results suggest that nearly half of the fish between the boards and more than 60 per cent of those in the path of the net are caught without the use of door-to-door ticklers. Dr R. R. Dickson gave an account of the ARL sector scanner which he has used in studies of gravel dredging. It is possible to pick up a dredged trench only six inches deep and two or three feet wide at a range of 120 yards. Exploratory holes and exploited patches of gravel are easily detected; the sectorscanning image is so clear that different holes can be clearly identified and found again on subsequent cruises. The results suggest that the holes and trenches made by gravel dredging may not be filled in by natural turbulence and movement of the neighbouring seabed. There can be little doubt that the sector scanner is a research tool of the greatest importance.

The special lecture was given by Professor J. A. Kitching (University of East Anglia) who described the programme of ecological studies by staff and students from the Universities of Cork, Bristol, East Anglia and elsewhere who have worked in and around Loch Ine for more than thirty years. Dr G. C. Kearn (University of East Anglia) described his field and experimental search for the means by which the free-living larvae of Entobdella solei, a parasite of the common sole, find their way to their hosts. The sole is active at night but buries itself in the sand and remains stationary for most of the day. The eggs of the parasite, which fall to the bottom, hatch soon after daylight, and Dr Kearn has been able to show that they are attracted, presumably by a chemo-perceptive mechanism, to the skin of the sole. Another speaker from the University of East Anglia, Dr I. N. McCave, attempted a budget for the deposition of mud in the North Sea. His results emphasized the need for much more information about the volumes and rates of transport of sediments out of estuaries. It was apparent from the discussion that more needs to be discovered about the effects of dumping and sewage effluent ; these may not be inconsiderable sources of sediments on the floor of the seas.

\section{MOLECULAR GENETICS \\ UAA in Eukaryotes}

from our Cell Biology Correspondent

THE belief that the genetic code is universal to all prokaryotes and eukaryotes, even though almost all the direct evidence for particular codon assignments has come from experiments with Escherichia coli and its phages, is, of course, a cardinal tenet of molecular biologists. And the faithful will no doubt be reassured of the inviolability of their doctrines by what Stewart, Sherman, Jackson, Thomas and Shipman have to say in the current issue of the Journal of Molecular Biology $(67,83 ; 1972)$. This group, pursuing their penetrating and exemplary analysis of the molecular genetics of the cytochrome $c$ in baker's yeast, have now established that in this eukaryote, as in $E$. coli, the codon UAA acts as a nonsense codon terminating the synthesis of polypeptides.

Stewart et al.'s unequivocal evidence comes from a detailed investigation of the properties of strains carrying a particular mutant designated cyl 9 , and of a large set of revertant strains. Yeasts carrying the cyl-9 mutation contain the normal amount of iso-2-cytochrome $c$, but they contain less than 0.5 per cent of the normal amount of iso-1-cytochrome $c$. Clearly this mutation must either prevent the synthesis of this cytochrome or it must cause the very rapid degradation of this protein. This property of strains carrying the cyl-9 mutation, together with the fact that the cy 1-9 mutation is suppressible (Gilmore, Stewart and Sherman, 1971), is, of course, redolent of the properties of strains of $E$. coli carrying amber or ochre mutations in structural genes; and to establish that the cyl-9 mutation results in the generation of an ochre codon in the structural gene for iso-1cytochrome $c$ Stewart et al. have exploited methods made familiar by bacterial geneticists.

In essence Stewart et al. have isolated spontaneous and induced revertants of cyl-9 mutant strains and have determined the amino-acid composition and amino-terminal sequence of the cytochromes they specify. By virtue of their composition the iso-1-cytochrome $c$ molecules made by the revertant strains fall into five classes; those with the normal composition and those with one glutamic acid residue replaced by either one lysine, serine, leucine or tyrosine residue. Analysis of peptide maps of cytochromes of these five classes reveals that the glutamic acid that has been substituted is the second residue in the polypeptide chain immediately following the amino-terminal threonine residue. Furthermore, the chymotryptic

\title{
Viral Messengers and Poly A
}

Although it would be erroneous to make the generalization that all eukaryotic messenger RNA molecules have at their $3^{\prime}$ ends a run of between 100-200 adenylic acid residues--putative histone messengers do not-the tally of mRNAs known to have such a $3^{\prime}$ poly A sequence grows almost daily. In Nature New Biology next Wednesday (July 26), for example, Weinberg, Ben Ishai and Newbold and Eaton, Donaghue and Faulkner report respectively the presence of poly A tracts in SV40 and Sindbis virus messengers.

Weinberg et al. find that SV40 messengers in cytoplasmic polysomes in permissive, monkey BSC1 cells during replication of the virus terminate in a tract of about 150-200 bases, at least 90 per cent of which are adenylic acid. This tract must be added to the messengers after transcription because the viral DNA does not contain more than twenty consecutive $\mathrm{A}=\mathrm{T}$ base pairs. Likewise in non-permissive mouse 3T3 cells transformed by SV40 cytoplasmic messengers containing sequences specified by the resident transforming SV40 genome terminate in a similar poly $A$ tract. Furthermore, in infected cells heterogeneous nuclear RNA molecules containing viral sequences and believed to be precursors of the viral messengers found in cytoplasmic polysomes also terminate in a $3^{\prime}$ poly A tract. It seems therefore that the cellular enzyme(s) in the nucleus responsible for adding poly A to messenger precursor molecules does not distinguish between RNAs specified by the cell and the viral genomes.

Sindbis virus, unlike SV40, has a single stranded RNA genome, and in extracts of infected cells two major and several minor species of viral RNA have been identified by gel electrophoresis. To obtain Sindbis mRNAs Eaton et al. isolated polysomes from infected cells, dissociated them and disrupted the ribonucleoprotein that was released to leave naked RNA which they analysed by centrifugation through sucrose gradients. These viral RNAs sediment at $4 \mathrm{~S}, 16-28 \mathrm{~S}$ and $42 \mathrm{~S}$. Digestion of the 16-28S RNA by pancreatic and $T_{1}$ ribonuclease liberates a resistant fragment which sediments at 3-4S and contains 75 per cent adenylic acid.

Sindbis mRNAs, which are synthesized in the cytoplasm off an RNA template, thus seem to contain poly $A$ tracts. Whether these tracts are coded by the viral genome rather than added after transcription remains to be seen, although Eaton et al. suggest the former situation may well be the case. 